\title{
Influence des variables distales sur la perception des risques : une revue de la littérature de 1978 à 2005
}

\author{
Bruno CHAUVIN* et Danièle HERMAND** \\ * Laboratoire «Éducation, Cognition, Développement », Université de Nantes, France \\ ** Laboratoire « Temps, Émotion et Cognition », Université Charles de Gaulle - Lille 3, France
}

\begin{abstract}
Résumé : Cette recherche présente une revue de littérature concernant l'influence des variables distales sur la perception des risques. Deux grandes catégories de travaux ont été considérées : (a) Ceux consacrés à tester l'effet des variables sociodémographiques sur le risque perçu, à savoir les variables Genre, Age, Profession, Niveau d'expertise scientifique, d'instruction, de revenus, Orientation politique et Point de vue individuel concernant la cible visée; (b) Ceux centrés sur les déterminants psychosociaux du risque perçu, à savoir les variables Statut individuel dans la société, Exposition médiatique, Visions du monde, Anxiété, Confiance sociale, Croyances religieuses, et Personnalité. Malgré l'apport d'un double éclairage à la fois descriptif et explicatif, il reste encore une grande part de la perception des risques à comprendre.
\end{abstract}

« Les individus réagissent aux activités hasardeuses selon leurs perceptions des risques que ces activités posent. Ce qu'ils perçoivent, pourquoi ils le perçoivent de cette façon, et comment ils se comporteront ultérieurement sont des questions d'une grande importance pour les industries et les gouvernements qui tentent d'estimer et d'implanter de nouvelles technologies » (Peters et Slovic, 1996, pp. 1427-1428).

Nées dans les années 1960 au moment du débat sur le nucléaire, les recherches sur la perception des risques ont depuis connu un développement accru et sont aujourd'hui considérées comme un sujet d'un intérêt considérable à la fois pour les chercheurs et pour les décideurs politiques (Sjöberg, 1996a, 2000a). Avant tout, il convient de définir la perception des risques en spécifiant la signification de chacun de ces composants, à savoir le risque, la perception, et la perception des risques ${ }^{1}$. Le risque est par nature subjectif et représente un mélange de science et de facteurs psychologiques, sociaux, culturels, et politiques importants (Slovic, 1999). La perception [des risques] correspond à l'appréhension d'un vaste ensemble de critères subjectifs [associés au risque] (Sjöberg, 1996a, 1998c ; Slovic, 1999). Finalement, étudier la perception des risques signifie examiner les opinions que les individus expriment lorsqu'ils doivent, de diverses manières, évaluer des technologies, substances ou activités plus ou moins risquées (Slovic, Fischhoff et Lichtenstein, 1982).

De façon synthétique, il est apparu que la recherche sur la perception des risques a visé à répondre à deux questions principales : (a) Pourquoi certaines activités, substances, ou technologies sont-elles perçues plus risquées que d'autres par la population en général ?; (b) Pourquoi certains individus perçoivent-ils un ensemble d'activités, substances ou technologies plus risquées que d'autres individus?

Aujourd'hui, après trois décennies de recherche sur la perception des risques, il apparait que la réponse à la première question a été apportée de façon satisfaisante, principalement par le biais de l'application de la méthodologie du paradigme psychométrique (conçue par Slovic, Fischhoff et Lichtenstein, 1980, 1985). Cette stratégie de recherche originale - considérée aujourd'hui par de nombreux auteurs comme l'approche la plus convaincante pour caractériser la perception des risques (Boholm, 1998 ; Bronfman et Cifuentes, 2003; Cha, 2000, parmi d'autres) - consiste à recueillir des jugements quantitatifs d'individus sur le niveau de risque présenté par une vaste taxonomie d'activités, substances ou technologies, selon des échelles de cotation en 7 ou 11 points. Ces mêmes activités, substances, ou technologies sont ensuite jugées selon un ensemble de caractéristiques qualitatives supposées rendre compte de la perception des risques (volontarisme, peur, catastrophicité, ou encore contrôlabilité). Une

Pour toute correspondance relative à cet article, s'adresser à Bruno Chauvin, Laboratoire «Éducation, Cognition, Développement ", U.F.R. de psychologie, Université de Nantes, Chemin de la Censive du Tertre, Boîte Postale 81227, 44312 Nantes CEDEX, France ou par courriel à < bruno. chauvin@univ-nantes.fr>.

1.Étant acquis que la définition du risque est sujet à controverse (Fischhoff, Watson et Hope, 1984) et qu'aucune définition du risque correcte ou appropriée à tous les problèmes n'est atteignable (Cadet et Kouabenan, 2005), les définitions exposées dans cet article ne se veulent ni exhaustives, ni consensuelles, mais sont celles adoptées par une majorité de psychologues sociaux travaillant sur ce thème de la perception des risques. 
fois tous ces jugements effectués, ils sont corrélés et analysés factoriellement, ce qui permet de produire une structuration factorielle de la perception des risques - ou " carte cognitive » - qui synthétise les jugements obtenus en deux, trois ou quatre grands facteurs. C'est ainsi que Slovic et al. $(1980,1985)$ ou Mullet, Duquesnoy, Raiff, Fahrasmane et Namur (1993) ont mis en évidence que le sentiment de peur inspirée par un risque (premier facteur) et le manque de connaissance associée à un risque (deuxième facteur) étaient les déterminants principaux du risque perçu.

En revanche, apporter une réponse satisfaisante à la deuxième question est apparu moins évident ; les résultats des recherches ayant tenté d'y répondre ont été plus flous, plus imprécis, et restent aujourd'hui peu satisfaisants (Sjöberg, 2003). Ceci s'explique par le fait que caractériser les personnes est beaucoup plus difficile que caractériser les activités, substances ou technologies hasardeuses : la variabilité individuelle est considérable. En conséquence, les dimensions personnelles considérées ont largement varié d'une étude à l'autre, en fonction des préférences des auteurs.

L'objectif de cet article est donc d'éclaircir ce pan de la recherche sur la perception des risques, en proposant une revue de littérature consacrée aux études qui ont tenté de comprendre - c'est-à-dire décrire et/ou expliquer - pourquoi certains individus perçoivent un ensemble d'activités, substances ou technologies plus risquées que d'autres individus. En d'autres mots, cet article rend compte des travaux qui se sont intéressé à l'influence des variables distales sur la perception des risques. Par opposition aux variables proximales - qui renvoient à des variables sémantiquement étroitement reliées au risque perçu (c'est-à-dire des variables caractéristiques du risque comme la peur ressentie face à un risque ou le manque de connaissance associé à un risque) - les variables distales ${ }^{2}$ sont sémantiquement éloignées et distantes du risque perçu, et renvoient plutôt à des caractéristiques individuelles sociodémographiques (par exemple, le genre ou l'âge) ou psychosociales (par exemple, les croyances ou la personnalité).

Dans cette optique, deux grandes catégories de recherches ont été considérées. La première catégorie - présentées dans la première partie de cette revue de littérature - a regroupé tous les travaux descriptifs qui ont testé simplement l'effet des variables individuelles distales dites sociodémographiques sur la perception des risques. Ces travaux - dont le but a été d'évaluer si les estimations effectuées par tel type d'individus étaient significativement différentes de celles effectuées par tel autre type d'individus - ont considéré les variables Genre, Âge, Niveau d'expertise scientifique, Niveau d'instruction, Profession, Niveau de revenus, Orientation politique et Point de vue adopté par l'individu quant à la cible visée par le risque (risque considéré pour soi, pour les autres, ...). Bien qu'intéressantes à un niveau descriptif, ces recherches ne fournissent pas d'explications quant au niveau de risque perçu, puisqu'elles ont principalement visé à constater l'existence ou non de différences entre les individus, sans envisager les raisons de ces différences. Ainsi, de façon complémentaire à cette première série de recherches, une seconde catégorie d'études - développées dans la deuxième partie de cette revue de littérature - a regroupé tous les travaux à tendance explicative qui se sont centré sur les déterminants individuels distaux psychosociaux du risque perçu. Ces travaux - dont le but a été d'identifier les variables psychosociales contribuant à expliquer une certaine partie de la perception des risques - ont considéré les variables Statut/Position dans la société, Exposition médiatique, Visions du monde, Anxiété, Confiance sociale, Croyances religieuses, et Personnalité ${ }^{3}$. De façon supplétive à celles de la première catégorie, les recherches de cette seconde catégorie permettent une caractérisation plus optimale du risque perçu par les individus, puisqu'elles ont mis à jour une partie des raisons probables des différences individuelles au niveau du risque perçu, même s'il reste encore une grande part de la perception des risques à expliquer.

\section{Effet des variables sociodémographiques sur le risque perçu}

Effet du genre

Selon Slovic (1999), plusieurs douzaines d'études ont montré que les hommes tendaient à juger les risques de façon plus faible et moins problématique que les femmes.

Bastide, Moatti, Pages et Fagnani (1989) ont montré que les femmes en France sont plus « averses » que les hommes aux risques technologiques et industriels

2. Les termes «proximal » et « distal» ont été introduits par Sjöberg (2003).

3.Pour chacune de ces variables comme pour celles de la première catégorie de recherches présentée, un ordre chronologique ascendant a été adopté, allant de la recherche la plus ancienne à la recherche la plus récente. 
posés par les usines chimiques ou nucléaires, les raffineries, et même les centrales hydroélectriques.

Savage (1993) - visant à étudier précisément les influences démographiques sur les perceptions des risques dont celle du genre - a mis en évidence à partir d'un échantillon de Chicago que les femmes ressentaient être plus exposées et avoir plus peur des risques représentés par les accidents d'avions commerciaux, les feux dans les maisons, les accidents automobiles, et les cancers de l'estomac que les hommes.

Une étude de Karpowicz-Lazreg et Mullet (1993) a identifié la perception des risques d'étudiants français masculins et féminins. Une partie des risques étudiés a donné lieu à des différences de perception entre les hommes et les femmes. Pour ces risques, la perception des risques des femmes a été à chaque fois plus élevée que celle des hommes.

Zhang (1994) a étudié la façon dont un échantillon de Chinois percevait 20 risques environnementaux menaçant la société chinoise dans son ensemble. Il a montré que le niveau de risque perçu par les femmes était légèrement supérieur à celui perçu par les hommes.

Cohn, Macfarlane, Yanez et Imai (1995), dans leur étude de la perception des risques d'adolescents et de parents américains, ont montré qu'ils existaient des différences de genre parmi les adolescents. Les perceptions des risques des garçons sont plus faibles que celles des filles, concernant des risques associés à des participations expérimentales, occasionnelles et fréquentes à des activités à risques.

Dans une étude de Slovic, Malmfors, Krewski, Mertz, Neil et Bartlett (1995), il est apparu que des femmes toxicologues ont estimé les items comme étant modérément ou hautement risqués plus fréquemment que les hommes.

Sjöberg, Kolarova, Rucai et Bernström (1996) ont étudié la perception des risques en Bulgarie et en Roumanie. 240 bulgares et 192 roumains de diverses professions ont estimé 100 items relatifs à des activités humaines, des substances chimiques et des technologies, selon le risque de mort qu'ils représentaient pour l'ensemble de la société. Alors qu'en Bulgarie les différences de genre ont été faibles, de grandes différences entre les estimations des hommes et des femmes ont été mises en évidence en Roumanie : les estimations des femmes ont globalement été supérieures à celles des hommes.
Schütz et Wiedemann (1998) ont examiné les jugements des risques associés aux produits de consommation. 220 femmes et 188 hommes de nationalité allemande ont évalué 30 produits de consommation selon le risque personnel et le risque pour l'environnement. Pour le risque personnel, les jugements des femmes ont été plus élevés que ceux des hommes pour la plupart des produits. Concernant le risque pour l'environnement, les jugements des femmes ont eu tendance à être supérieurs à ceux des hommes pour la plupart des produits.

Lai et Tao (2003) ont travaillé sur la perception de Chinois de Hong-Kong à propos de risques environnementaux. 167 chinois de Hong-Kong (48\% d'hommes et $52 \%$ de femmes) ont estimé 25 items selon leur degré de risque. Considérés localement ou globalement, les risques perçus par les femmes ont été plus élevés que ceux perçus par les hommes.

Une étude de Xie, Wang et Xu (2003) a notamment examiné les différences de perception des risques à propos de diverses questions dans la Chine actuelle entre des hommes et des femmes salariés. À partir des estimations de 430 hommes et 376 femmes concernant 28 risques, il est apparu que la perception des risques des femmes a été à chaque fois plus élevée que celles des hommes.

Ainsi, même si deux études - respectivement suédoise et égyptienne (Ahmed, Macri et Mullet, 2006 ; Källmén, 2000) - ont mis en évidence une absence de différences entre les hommes et les femmes, il semble qu'en accord avec la déclaration de Slovic (1999), la très grande majorité des études menées depuis une quinzaine d'années à travers de nombreux pays a montré que la perception des risques des femmes était plus élevée que celles des hommes.

\section{Effet de l'âge}

Selon Hermand, Mullet et Rompteaux (1999), l'effet de l'âge a fait l'objet d'un nombre plus restreint d'études, qui n'ont pas réussi à mettre en évidence une tendance nette concernant l'effet de l'âge sur le risque perçu. Un certain nombre de ces études est présenté ci-dessous.

Savage (1993) a montré que les plus jeunes personnes se sentent plus exposées et ont plus peur des risques posés par les accidents d'avions commerciaux, les feux dans les maisons, et les accidents automobiles que les plus vieilles personnes. 
Sjöberg et Torell (1993) ont étudié la façon dont se développaient l'acceptation et l'évaluation morale des risques. Un échantillon de 212 élèves âgés de 10 ans $(n=70), 13$ ans $(n=87)$, et 16 ans $(n=65)$, comptant autant de garçons que de filles, a participé à cette étude. Globalement, une attitude plus indulgente (évaluation morale des risques plus positive et acceptabilité plus grande) se développe au fur et à mesure que l'enfant grandit.

S'intéressant à la perception des risques environnementaux vus par des chinois âgés de 17 à 55 ans, Zhang (1994) a mis en évidence que les personnes plus âgées étaient plus inquiètes à propos de risques qui menacent les biens et la vie que les plus jeunes.

Cohn et al. (1995) se sont penché sur la perception des risques exprimée par 376 adolescents américains (dont l'âge moyen était de 15,2 ans) et 160 adultes parents de ces adolescents (dont l'âge moyen était de 41 ans). Les résultats ont suggéré que les perceptions des risques des parents et des adolescents étaient différentes : les adolescents - vraisemblablement par échec à percevoir les risques - ont estimé une majorité des 14 activités à juger comme significativement moins risquées que leurs parents, que ce soit pour des participations expérimentales, occasionnelles, ou fréquentes.

Benthin, Slovic et Severson (1993) ont examiné la façon dont 41 participants âgés en moyenne de 15,5 ans ont estimé 30 risques. Ils ont montré que les adolescents « se déclarant participants »ont reporté moins de peur, moins de risque personnel, moins de risque pour les pairs, ou encore moins d'effets graves que les adolescents « non participants ».

Hermand et al. (1999) ont étudié la perception des risques parmi plusieurs groupes d'âge différent. 160 individus répartis en cinq groupes d'âge (enfants, adolescents, jeunes adultes, adultes matures et personnes âgées) ont coté une liste de 91 items selon le niveau de risque en France représenté par chacun d'eux. Malgré la similitude au niveau de l'ordonnancement et la proximité des moyennes (toutes comprises entre 30 et 35 sur 100), un effet significatif de l'âge a été observé : les estimations des enfants ont été significativement supérieures à celles des autres groupes d'âge et les estimations des jeunes adultes ont été significativement inférieures à celles des autres groupes.

Dans une étude de Lai et Tao (2003), 167 Chinois de Hong-Kong, âgés de 18 à 63 ans, ont estimé 25 risques environnementaux selon leur degré de menace à un niveau local et à un niveau global. Les résultats ont indiqué que les participants plus âgés ont perçu les risques comme plus menaçants pour l'environnement local et global que les participants plus jeunes.

Récemment, Ahmed et al. (2006) ont examiné la perception des risques d'égyptiens. 206 étudiants jeunes adultes - âgés de 17 à 25 ans - et 224 adolescents - âgés de 12 à 16 ans - ont estimé 141 items selon le risque qu'ils représentaient. Les estimations des adolescents et des adultes ont été très proches en termes d'ampleur (moyennes non significativement différentes autour de 50 sur 100) et en termes d'ordonnancement.

Ainsi, toutes ces études ne permettent pas de mettre en évidence une tendance nette concernant l'effet de l'âge sur le risque perçu. Certaines d'entre elles ont mis en évidence que les plus jeunes avaient une perception des risques moins élevée que les plus âgés (par exemple, Cohn et al., 1995 ; Lai et Tao, 2003 ; Zhang, 1994); à l'inverse, d'autres ont montré que les plus jeunes avaient une perception des risques supérieure à celle des plus âgés (par exemple, Hermand et al., 1999 ; Savage, 1993). Enfin, Ahmed et al. (2006) ont mis en évidence une absence de différence nette entre plus jeunes et plus âgés. Sur la base des suppositions de Zhang (1994), ces différences de résultats concernant l'effet de l'âge sur le risque perçu pourraient provenir du type de risques étudiés.

\section{Effet du niveau d'expertise scientifique}

De très nombreuses recherches se sont intéressé à l'influence du niveau d'expertise scientifique sur le risque perçu, en comparant les perceptions émises par des individus dits « experts » (ingénieurs, toxicologues, ...) à celles de profanes (Sjöberg, 1998a; Slovic, 1992).

En fait, dès les premières études de la perception des risques (Slovic, 1987; Slovic et al., 1979, 1980, 1985), Slovic et ses collaborateurs ont mis en évidence une perception des risques différente de la part des experts comparée aux profanes. Selon Slovic (1999), ou Sjöberg (1996a), il existe une dichotomie saillante entre les experts et le public. Les experts sont vus comme fournissant des estimations des risques décrites comme objectives, analytiques, sages, et rationnelles. À l'inverse, le public est vu comme se fiant à des perceptions des risques subjectives, souvent hypothétiques, émotionnelles, imprudentes, et irrationnelles, déterminées par un ensemble de caractéristiques qualitatives. 
Kraus, Malmfors et Slovic (1992) ont illustré cette différence de perception des risques experts vs profanes selon une perspective intitulée "toxicologie intuitive ». Leurs données américaines ont montré des différences substantielles dans les attitudes et croyances des experts comparées à celles des profanes. Précisément, les individus « tout venant» tendent à penser que toute exposition à une substance nocive ou à un cancérigène toxique - peu importe la dose - a des chances de se révéler dangereuse. En revanche, pour les toxicologues, les dommages provoqués par un produit toxique dépendent fortement de la dose d'exposition.

Slovic et al. (1995) ont étendu ces résultats à partir de données canadiennes. Dans leur étude, 150 membres de la Société Canadienne de Toxicologie et 1506 profanes ont indiqué le degré de risque pour la santé du public canadien qu'ils associaient à un ensemble d'items variés. Globalement, les membres $\mathrm{du}$ public ont eu une perception des risques beaucoup plus élevée que les toxicologues, en utilisant l'intitulé « hauts risques » plus fréquemment que les toxicologues pour 36 des 38 items considérés.

Slovic (1996) a révélé que la perception des risques liés à la radiation fournie par le public est très différente de celle fournie par les experts. Dans certains cas, les perceptions du public sont extrêmement plus élevées que celles des experts (c'est le cas notamment pour l'énergie nucléaire); dans d'autres cas, elles sont beaucoup moins élevées que ce que pensaient les experts (par exemple, c'est le cas pour les rayons $\mathrm{X}$ en radiologie).

McDaniels, Axelrod, Cavanagh et Slovic (1997) ont étudié la perception des risques écologiques relatifs aux environnements aquatiques. Pour environ un tiers des 33 risques écologiques considérés, les estimations des experts ont été significativement différentes de celles des profanes.

Mullet, Ben Bouazza, Dupont et Bertrand (1998) ont étudié la perception des risques associés à la production d'énergie. 80 étudiants universitaires français ont estimé 115 items combinant des sources d'énergie et des phases du processus de production/distribution d'énergie. Certains de ces jugements ont été comparés à ceux de 8 experts ingénieurs en charge de la sécurité de centrales nucléaires. En ce qui concerne le nucléaire, les estimations moyennes des experts ont été généralement inférieures à celles des étudiants d'environ 10 points sur 100. En revanche, concernant les sources d'énergie relatives à l'eau, au vent, et au soleil, les estimations des experts ont été similaires à celles des étudiants.
Sjöberg (1998c) a comparé de façon systématique la perception des risques d'experts à celle d'un public suédois. Il a distingué trois types de risques : (a) Dans le cas de risques ordinaires et bien connus, le public semble faire des jugements moyens plutôt corrects et en accord avec ceux des experts ; (b) Dans le cas de risques professionnels et associés au style de vie (comme le radon domestique), alors que les experts sont préoccupés, le public est peu inquiet et souvent passif; (c) Dans le cas des risques technologiques (comme ceux associés au nucléaire), les risques sont jugés par les experts comme étant très faibles, alors que les membres du public ressentent qu'ils sont élevés. Selon l'auteur, un cas qui a été plutôt bien examiné est celui de la destruction de déchets nucléaires (Barny, Bonnefous et Brenot, 1992).

Récemment, Lazo, Kinnell et Fisher (2000) ont comparé les perceptions d'experts et de profanes à propos de risques pour l'écosystème, et plus particulièrement à propos de risques issus du changement climatique global. Un groupe d'experts (10 professeurs et chercheurs spécialistes des questions d'écologie et 16 employés experts de l'Association de Protection Environnementale Américaine) et un groupe des 246 profanes ont estimé 25 risques selon 31 échelles de jugement. Globalement, les profanes ont estimé les risques comme étant plus sévères et moins acceptables que les experts.

Toutes ces études montrent une différence de perception des risques entre les experts et les profanes, les derniers percevant la plupart du temps plus de risques que les premiers. Selon Slovic (1992), ce résultat a suscité des réactions très sévères envers les profanes de la part de certains observateurs. Un psychiatre (Dupont, 1980) a par exemple émis l'idée que la peur irrationnelle des centrales nucléaires était basée sur des estimations erronées des risques; Un physicien nucléaire et un ardent défenseur de l'énergie nucléaire ont même prétendu que «le public avait été rendu fou à propos de la peur des radiations » (Slovic, 1992, p. 150). Pourtant, la recherche sur la perception des risques montre que la dialectique « irrationalité des profanes vs rationalité des experts » énoncée par ces affirmations est illégitime. D'abord, parce que la conceptualisation basique des risques de la part des profanes n'est pas irrationnelle. Beaucoup plus riche que celle des experts, elle prend notamment en compte les bénéfices directs associés aux technologies ou encore la nature des individus promouvant telle ou telle technologie, et reflète ainsi des inquiétudes légitimes typiquement omises par les experts (Slovic 1992, 1996). Ensuite, parce que les jugements de 
risques formulés par les experts peuvent eux aussi s'avérer biaisés. À l'image des profanes, même les individus les plus formés sont influencés par le contexte spécifique dans lequel s'inscrivent les questions de risques, particulièrement quand ils sont forcés d'aller au-delà des limites des données disponibles et donc de faire confiance à leur intuition (Finucane, Slovic, Mertz, Flynn et Satterfield, 2000a ; Freudenburg, 1988 ; Slovic et al., 1980).

\section{Effet du niveau d'instruction/formation}

Toutes les recherches qui se sont directement intéressé à l'effet du niveau d'instruction sur le risque perçu sont arrivées à la même conclusion: les individus moins instruits perçoivent globalement plus de risques que les individus mieux instruits.

C'est notamment le cas de l'étude de Pilisuk et Acredolo (1988) - menée à partir d'un échantillon aléatoire de 450 personnes de Californie - qui a montré que les plus grandes inquiétudes concernant des risques technologiques étaient exprimées par les individus moins instruits par rapport à ceux mieux instruits.

Savage (1993) s'est aussi penché sur ce sujet et a montré que les personnes ayant un niveau d'instruction plus faible ressentent plus de peur et se sentent plus exposées personnellement aux risques que ceux ayant un niveau d'instruction plus élevé.

Une autre recherche traitant de cet effet a été conduite par Lai et Tao (2003) à partir d'un échantillon de Chinois de Hong-Kong. Deux groupes de participants ont été formés selon leur niveau de formation « élevé " vs « faible». Les résultats ont indiqué que les participants moins formés ont perçu les risques comme étant plus menaçants pour l'environnement local que ne l'ont fait les participants mieux formés.

\section{Effet de la profession}

L'effet de la profession sur le risque perçu est relativement bien documenté. Parmi les études qui s'y sont intéressé, celle de Bastide et al. (1989) a montré que les cols bleus et les cols blancs à faibles revenus avaient une plus forte aversion pour les risques de type industriel que les cols blancs à hauts revenus.

Nyland (1993) a étudié et comparé la perception des risques d'échantillons brésiliens et suédois. Neuf échantillons différents (des habitants de taudis brésiliens, des infirmières suédoises et brésiliennes, des travailleurs peu qualifiés suédois et brésiliens, des étudiants universitaires en économie suédois et bré- siliens, et des étudiants diplômés ingénieurs suédois et brésiliens) ont notamment estimé 100 items selon le risque de mort qu'ils représentaient pour l'ensemble de la société. Au Brésil comme en Suède, la perception des risques la plus élevée a été celle des infirmières (et des habitants de taudis brésiliens) et la moins élevée a été celle des étudiants diplômés ingénieurs.

Suivant la même procédure, Sjöberg et al. (1996) ont examiné la perception des risques en Bulgarie et en Roumanie. Six sous-échantillons par pays (étudiants en économie, étudiants ingénieurs, infirmières, enseignants, ouvriers et Sans Domicile Fixe) ont notamment estimé 100 items selon le risque de mort qu'ils représentaient pour l'ensemble de la société. En Bulgarie comme en Roumanie, la perception des risques la plus élevée a été celle des infirmières et la moins élevée a été celle des étudiants diplômés ingénieurs (égale à celle des enseignants pour la Roumanie).

Dans leur étude de la perception des risques de Chinois, Xie et al. (2003) ont montré que la diversité des professions et du statut d'emploi des individus avait un effet sur le risque perçu. En effet, malgré quelques similitudes, les perceptions des risques exprimées par des fonctionnaires et/ou employés, des directeurs et/ou gérants, des personnels et/ou ouvriers, des professeurs, et des étudiants se sont avérées être globalement différentes.

Ainsi, il semble que le rôle professionnel et l'expérience jouent un rôle important dans la perception des risques. La confrontation plus ou moins fréquente à certains risques dans le contexte professionnel apparaît influencer la perception des risques.

\section{Effet du niveau de revenus}

Selon Vaughan et Nordenstam (1991), l'effet du niveau de revenus sur le risque perçu a été peu directement étudié car il a eu tendance à être confondu avec ceux du niveau d'instruction et/ou de la profession. Toutefois, d'après les travaux qui ont testé cet effet, il apparaît que les individus les plus pauvres ont les perceptions des risques les plus élevées.

Par exemple, Pilisuk et Acredolo (1988) ont trouvé que les plus grandes inquiétudes pour les risques technologiques étaient exprimées par les individus les plus pauvres, ayant moins les moyens financiers de se défendre.

De façon similaire, Savage (1993) a montré que les personnes aux revenus les moins élevés ressentent 
globalement plus de peur et plus d'exposition personnelle aux risques que les individus aux revenus plus élevés.

\section{Effet de l'orientation politique}

Un certain nombre de recherches a montré des effets de l'orientation politique sur la perception des risques, et notamment sur le risque perçu associé au nucléaire.

Parmi elles, il y a l'étude de Bastide et al. (1989), dont l'objectif a été de rendre compte de la relative acceptabilité sociale des technologies en France comparée aux conflits que ces technologies génèrent en Allemagne. Ces auteurs ont conduit 1000 interviews personnelles auprès de Français à propos des risques posés par diverses technologies et ont comparé leurs résultats à ceux d'interviews effectuées sur un échantillon allemand. Ces comparaisons ont notamment permis de mettre en évidence une différence de risque perçu selon l'orientation politique : alors que les individus affiliés aux puissants partis de gauche allemands ont exprimé une forte aversion pour les risques industriels, les individus affiliés aux puissants partis de droite conservateurs français ont minimisé les risques industriels.

Eiser, Hannover, Mann, Morin, Van Der Pligt et Webley (1990) ont étudié les perceptions des risques nucléaires formulées par des individus provenant de pays plus ou moins éloignés de Chernobyl

- à savoir l'Australie (Adélaïde), le Royaume-Uni (Exeter), la France (Aix-en-Provence), les Pays-Bas (Amsterdam), et l'Allemagne (Berlin) - et les ont mises en relation avec des mesures de préférences politiques. Globalement, dans chacun des 5 groupes, ceux qui avaient les visions politiques les plus conservatrices - autrement dit les plus ancrées «à droite »- étaient aussi ceux qui apparaissaient les plus favorables au nucléaire.

La recherche de Wiegman, Gutteling et Cadet (1995) a comparé la perception de l'énergie nucléaire et du charbon en France et aux Pays-Bas. Selon ces auteurs, le résultat selon lequel les français expriment une attitude plus négative envers l'énergie nucléaire, une plus grande probabilité de risques et une plus forte intention d'atténuer ces risques peut s'expliquer par une politique gouvernementale plus autoritaire, centrale et bureaucratique en France comparée aux Pays-Bas.

Une étude originale conduite par Sjöberg (1996b) a comparé la perception des risques de politiciens locaux responsables de la santé et de l'environnement à celle d'un vaste échantillon public de Suédois. Globalement, les deux échantillons ont exprimé une perception des risques très similaire. Toutefois, concernant les risques relatifs aux déchets nucléaires, les jugements des politiques ont été nettement plus faibles que ceux du public suédois.

Les orientations politiques semblent donc jouer un rôle sur le risque perçu. Toutefois, ce rôle est à relativiser car - à ce jour - il n'a été mis en évidence que sur un seul type de risques, ceux associés aux technologies énergétiques (principalement l'énergie nucléaire).

\section{Effet du point de vue adopté par l'individu quant à la cible visée ou effet de la cible}

« La cible du risque est un paramètre important dans les études sur le risque. Ce fait relativement simple ne semble pas être connu de tous, et de nombreuses études travaillent toujours sans spécifier les cibles du risque. Ceci indique que certains chercheurs oublient probablement le besoin de comprendre le risque personnel perçu, et ceci introduit de l'incertitude quant à la cible qu'ils étudient réellement » (Sjöberg, 2000a, p. 9).

Weinstein peut être considéré comme le premier auteur à avoir mis en évidence un effet de la cible sur le risque perçu. Cet auteur a étudié (a) des estimations effectuées par des étudiants sur leurs probabilités d'expérimenter des événements positifs et négatifs par rapport aux probabilités d'autres étudiants (Weinstein, 1980), et (b) des estimations du même type mais effectuées par un échantillon représentatif de la population américaine (Weinstein, 1987). Les résultats obtenus ont montré que les individus estimaient leurs chances d'être confrontés à des événements positifs être plus fortes que la moyenne et leurs chances d'être confrontés à des événements négatifs être inférieures à la moyenne, cette dernière tendance augmentant quand les individus pensaient contrôler ces événements («nuance » récemment confrirmée par Causse, Kouabenan et Delhomme, 2004 ; Sjöberg, 2000a) et s'atténuant quand ils avaient fait l'expérience personnelle de ces événements ("nuance » récemment confirmée par Weinstein, Lyon, Rothman et Cuite, 2000). Étant donné que Weinstein n'a travaillé que sur deux types de cibles, le risque pour soi et le risque pour les autres, il n'a pas parlé d'influence du type de cible. Plutôt, il a nommé cet effet « optimisme irréaliste» ou «biais d'optimisme », en référence à la tendance des individus à se croire moins sujets à risques que d'autres dans la même situation - tendance non limitée à l'âge, au genre ou à la profession, et souvent intro- 
duite quand les individus extrapolent à partir de leur manque d'expérience passée pour prédire leur (in)vulnérabilité future.

Depuis ces travaux princeps de Weinstein (1980, 1987), de nombreux auteurs ont confirmé et étendu l'existence de ce biais d'optimisme à de nombreuses cibles. Cette extension a entraîné un glissement de l'intitulé « optimisme irréaliste» vers l'intitulé « effet de la cible ».

Parmi ces auteurs, il y a Neil, Slovic et Hakkinen (1993). Ils ont introduit 4 cibles dans leur étude américaine sur le risque perçu posé par 47 substances chimiques ménagères : risque (a) pour les adultes, (b) les jeunes enfants, (c) les personnes âgées, et (d) l'environnement. Les résultats ont montré que les réponses données pour les trois cibles humaines ont été très similaires en termes d'ordonnancement. Par contre, entre ces trois séries de réponses et celles pour la condition » environnement », l'ordonnancement des risques a été différent.

Frewer, Shepherd et Sparks (1993) ont considéré une large gamme de risques alimentaires selon 3 cibles : le risque personnel, le risque pour d'autres et le risque pour la société. Les résultats ont indiqué que la perception des risques pour soi était inférieure à celles pour les autres ou pour la société, quelles que soient les caractéristiques des risques.

Nyland (1993) a étudié et comparé la perception des risques d'échantillons brésiliens et suédois. 29 items ont été jugés selon le risque personnel et 33 items ont été estimés selon le risque pour la société dans son ensemble. Dans ces deux pays, la perception des risques pour la société a été significativement supérieure à la perception des risques individuels.

Dans leur étude conduite en Allemagne, Schütz et Wiedemann (1998) se sont intéressé aux risques personnels et environnementaux posés par 30 produits de consommation. Malgré des jugements globaux similaires, les comparaisons entre les estimations du risque personnel et du risque pour l'environnement effectuées au sujet du même item ont montré de nombreuses différences. Pour la moitié des items, le risque pour l'environnement a été estimé plus élevé que le risque personnel; Pour l'autre moitié des items ( 12 items), ça a été l'inverse.

Källmén (2000) - dans une étude où 59 étudiants suédois ont estimé le risque personnel et le risque global associé à 34 items - a répliqué le résultat selon lequel le risque global est plus élevé que le risque personnel, même si la source de risque est la même.
La même année, Sjöberg (2000a) a lui aussi mis en évidence un effet de la cible sur le risque perçu, même s'il l'a interprété comme étant un déni du risque. À partir des estimations de 15 risques selon 3 cibles, l'auteur a en effet constaté que les estimations concernant les personnes en général étaient bien plus élevées que celles concernant la famille ou soi-même.

Orton, Sjöberg, Jung, Urge-Vorsatz et TamassynéBiro (2001) ont étudié les jugements de radiographistes industriels hongrois et anglais à propos des risques personnels et globaux posés par des sources nucléaires et non nucléaires. La tendance à s'estimer moins à risque que les autres est apparue exister et être significative pour les deux groupes.

La recherche menée par Lai et Tao (2003) sur les perceptions de Chinois de Hong-Kong des risques environnementaux posés par 25 sources à un niveau local et à un niveau global a indiqué que, globalement, les risques ont été perçus comme étant significativement plus menaçants pour l'environnement global que pour l'environnement local.

En plus d'avoir eux aussi constaté un effet de la cible sur le risque perçu, Hermand, Karsenty, Py, Guillet, Chauvin, Simeone, Munoz Sastre et Mullet (2003) ont apporté des précisions quant à la nature des risques touchés par la cible. Leurs résultats ont en effet montré que l'effet de la cible a été négligeable pour tous les risques associés aux activités ordinaires, aux transports urbains, aux activités d'extérieur, et aux soins médicaux, mais qu'il a été important pour les risques relatifs aux polluants et à la déviance sexuelle et addictive : dans la condition « risque pour les êtres humains en général », les participants ont coté ces risques de manière supérieure à la condition « risque pour les français », et dans la condition « risque pour les français », les participants ont coté ces risques de manière supérieure à la condition « risque personnel». Ce dernier résultat selon lequel l'effet de la cible varie d'un domaine de risque à l'autre a récemment été confirmé par Bronfman et Cifuentes (2003).

Très récemment, Kobbeldvedt, Brun et Laberg (2004) ont montré que les risques personnels de bles- sures perçus par des élèves officiers à propos de 28 incidents potentiels en situation militaire ont été estimés être significativement plus faibles que les risques globaux.

Menés dans différents pays et à propos de risques très variés, tous ces travaux montrent clairement que, selon le point de vue adopté par les individus 
quant à la cible visée, les jugements des risques sont différents.

La première catégorie d'études qui vient d'être présentée apporte un premier éclairage de type descriptif au problème que représente la compréhension de la perception des risques. En effet, l'éventail des travaux évoqués ci-dessus - en testant l'effet d'un ensemble de variables individuelles distales de nature sociodémographique sur la perception des risques - a permis de mettre en évidence l'existence de différences au niveau du risque perçu entre les individus, selon leur genre, leur âge, ou encore leur niveau d'expertise scientifique.

Cependant, ces recherches sont insuffisantes pour comprendre de manière optimale la perception des risques, puisqu'elles n'ont pas fourni d'arguments de type explicatif quant aux raisons potentielles à l'origine de ces différences. C'est pourquoi la deuxième partie de cette revue de question expose une seconde catégorie de travaux qui regroupe les études ayant eu pour objectif d'identifier des déterminants individuels distaux de nature psychosociale $\mathrm{du}$ risque perçu. Les déterminants développés ont été relatifs au statut ou position des individus dans la société, à leur exposition médiatique, à leurs visions du monde, à leur degré d'anxiété, à la confiance sociale qu'ils accordent aux institutions d'expertise et de gouvernement, aux croyances auxquelles ils adhèrent, et enfin à leur personnalité.

\section{Influence des variables \\ psychosociales sur le risque perçu}

Influence du Statut / Position des individus dans la société

Présenter les travaux qui ont montré l'influence du statut/position des individus dans la société sur le niveau de risque perçu revient à évoquer le cheminement suivi par ces travaux, étant donné que ce déterminant n'a pas été identifié de façon directe mais plutôt pas à pas, par « essai-erreur ».

D'après Slovic (1999), les premières propositions tentant de rendre compte des différences de perception entre les hommes et les femmes ont été les suivantes : si les femmes perçoivent davantage de risques que les hommes, c'est dû à (a) un facteur biologique : elles donnent naissance (Steger et Witt, 1989) ; (b) un facteur sociologico-historique ou culturel : elles sont socialisées pour éduquer et maintenir la vie alors que les hommes sont fournisseurs économiques (Steger et Witt, 1989); (c) un facteur personnologique: elles sont plus prêtes que les hommes à exprimer ouvertement leurs inquiétudes (Fiske, 1987); (d) un facteur situationnel : les femmes sont plus souvent « victimes » que les hommes, ce qui augmente leur vulnérabilité perçue aux risques (Baumer, 1978); (e) un facteur éducationnel : les femmes ont un niveau d'instruction globalement plus faible que les hommes (Sjöberg et Drottz-Sjöberg, 1993).

Toutefois, insatisfaits de ces propositions, plusieurs auteurs ont mené des recherches les invalidant et/ ou les remplaçant par d'autres explications plus fondées (Slovic, 1999).

Parmi ces recherches, deux ont montré l'inanité du facteur éducationnel selon lequel les différences entre les hommes et les femmes sont dues à des différences de connaissance, d'instruction, et d'entraînement. Une première étude menée par Barke, Jenkins-Smith et Slovic (1997) a en effet mis en évidence que la perception des risques d'hommes membres d'une association américaine pour l'avancement de la science était moins élevée que celle de femmes membres de la même association. Une deuxième étude de Mertz, Slovic et Purchase (1998) a confirmé ce résultat et montré que les hommes membres de la Société Toxicologique Britannique étaient beaucoup moins enclins que les femmes toxicologues à juger les risques comme élevés. Dans ces deux cas, il est certain que ces femmes scientifiques ne peuvent pas être accusées de manquer de connaissances et d'alphabétisation technologique.

Une autre recherche - réalisée par Flynn, Slovic et Mertz (1994) - a non seulement permis d'invalider toutes les autres propositions évoquées ci-dessus mais a en plus procuré une explication bien plus fondée quant aux causes potentielles à l'origine des différences de perception des risques entre les hommes et les femmes. Après avoir estimé les risques pour les Américains associés à 25 items divers, les 1512 personnes de cette étude ont répondu à plusieurs questions d'ordre sociodémographique et d'ordre attitudinal. Les résultats ont montré que les hommes blancs ont fourni de façon consistante des estimations de risques plus faibles que celles des femmes blanches, des hommes non blancs et des femmes non blanches, ces trois sous-échantillons ayant donné des estimations globalement équivalentes. Dans le but de préciser ce premier résultat, les auteurs ont mis en évidence que les perceptions des risques les plus faibles avaient été exprimées par un sous-groupe de 246 hommes blancs, caractérisés par une meilleure instruction que les autres participants, de plus hauts revenus, une orientation politique plus conservatrice, moins d'inquiétudes pour les futures générations, et une plus grande confiance 
dans le gouvernement et l'industrie. Autrement dit, selon les auteurs, il existerait un effet du statut sur la perception et l'acceptation des risques. Comparés au groupe des hommes (blancs), les groupes des femmes (et hommes non-blancs) ont un statut dit « minoritaire ", tendent à avoir moins de contrôle, de pouvoir, et de bénéfices des technologies, et par conséquent perçoivent le monde comme plus risqué.

Les résultats de cette étude - depuis confirmés par Sjöberg (1998c), par Finucane et al. (2000a), ou par Palmer (2003) - ont donc permis (a) de remettre en cause toutes les propositions d'ordre biologique, sociologico-historique, personnologique et situationnelle, puisque les femmes blanches et les hommes non blancs ont une perception des risques similaire ; (b) de postuler et de confirmer l'existence d'un effet de statut sur la perception des risques, intitulé par les auteurs « effet homme blanc».

\section{Influence de l'exposition médiatique}

«Bien que les hommes vivent dans un monde aux multiples facettes, ils partagent en partie les mêmes inquiétudes. La modernisation et les nouveaux systèmes d'information [peuvent en partie expliquer cette équivalence dans les inquiétudes], dans le sens où les nouvelles technologies et les nouveaux modes de vie, de pensée ou de perception sont rapidement et globalement disséminés à travers le monde » (Boholm, 1998, p. 145).

Plus synthétiquement, selon Sjöberg et al. (1996, p. 150), «l'information est la clé ».

À partir d'une hypothèse formulée par Lichtenstein, Slovic, Fischhoff, Layman et Combs (1978) postulant un effet des média sur les estimations biaisées des fréquences réelles d'événements potentiellement létaux (plus ou moins médiatisés), Combs et Slovic (1979) ont mené la première étude montrant l'influence des média sur le risque perçu. Précisément, ces auteurs ont mis en évidence l'existence d'une forte corrélation positive entre le contenu de deux journaux américains et la perception des risques. Les causes de mort souvent rapportées par les média - car spectaculaires et catastrophiques - ont été jugées plus fréquentes que d'autres pourtant identiques au niveau fréquence réelle; à l'inverse, les causes de mort rarement présentes dans les média - car ordinaires - ont été jugées moins fréquentes que d'autres pourtant identiques au niveau fréquence réelle. D'après les auteurs, les informations apportées par les média sur différents types d'acci- dents semblent être un bien meilleur prédicteur des perceptions des risques des individus que les chiffres officiels de mortalité.

Suite à cette étude princeps, plusieurs recherches ont testé et confirmé l'influence de l'exposition médiatique sur la perception des risques.

Dans leur étude de la perception des risques issus des champs électromagnétiques de 50-60 hertz dégagés par les lignes de transmission et les couvertures chauffantes, Granger Morgan, Slovic, Nair, Geisler, MacGregor, Fischhoff, Lincoln et Florig (1985) ont montré que l'introduction de brochures informatives relatives à ces technologies augmentait la perception des risques posés par ces dernières, perçues alors comme plus effrayantes ou moins connues de la science. Cette influence de l'exposition à des brochures informatives sur le risque perçu a par la suite été confirmée par Mac Gregor, Slovic et Granger Morgan (1994).

Une recherche de Blomkvist et Sjöberg (1987), citée par Nyland (1993), a analysé les contenus des média suédois ainsi que leur influence sur la perception des risques. Selon eux, les reportages d'accidents sont biaisés car les media sont orientés dans ce qu'ils choisissent de rapporter. À leur tour, les média biaisent les perceptions des risques et augmentent le sentiment d'insécurité latent.

Plus récemment, Koné et Mullet (1994) ont confirmé l'influence des média sur le risque perçu via la comparaison des jugements de risques effectués par un groupe d'étudiants français à ceux effectués par un groupe d'intellectuels burkinabés hautement diplômés et ayant un accès à de nombreuses revues françaises. En effet, ces deux échantillons - pourtant très différents en termes de géographie, économie, politique, et ethnie, mais équivalents en termes d'exposition médiatique - ont délivré des perceptions des risques similaires.

De très nombreuses autres études ont utilisé l'influence de l'exposition médiatique sur la perception des risques pour rendre compte de leurs résultats.

Lorsque Englander, Farago, Slovic et Fischhoff (1986) comparent la perception des risques d'Américains à celle de Hongrois et constatent des inquiétudes trop grandes des Américains - à propos de catastrophes improbables - et le manque d'inquiétude des Hongrois - concernant des risques sanitaires réels - ils expliquent leurs résultats en évoquant l'influence de l'exposition médiatique : 
« la plupart des reportages sur les risques sont relatifs à des risques hors Hongrie, amenant les hongrois à croire que les événements néfastes se passent ailleurs. À l'inverse, aux États-Unis, la plupart des problèmes rapportés arrivent à l'intérieur du pays » (ibid., p. 65).

Goszczynska, Tyszka et Slovic (1991) se sont aussi servi de l'influence des média pour expliquer les différences de perception des risques constatées entre leurs participants polonais et des hongrois. Alors qu'en Pologne l'accessibilité à l'information a toujours été forte du fait de l'existence d'une presse catholique indépendante et de plusieurs journaux non censurés, cela n'a jamais été le cas en Hongrie du temps du communisme pendant lequel les Hongrois étaient soumis à une forte restriction informationnelle.

Pour Hermand et al. (1999), la faiblesse des différences constatées entre les estimations des risques d'enfants, d'adolescents, d'adultes et de personnes âgées est due à leur accès similaire aux média : «si leur perception des risques n'avait pas été influencée par les média, mais formée principalement par les expériences de la vie de tous les jours, des différences beaucoup plus fortes entre les enfants, les adolescents et les adultes auraient été trouvées » (ibid. p. 13).

Orton et al. (2001) ont cité la même raison pour rendre compte de l'absence de différences de perceptions des risques exprimées par des radiographistes industriels hongrois et anglais (les hongrois ayant aujourd'hui un accès illimité aux média).

Deux études de Neto et Mullet (2000) ont elles aussi invoqué l'exposition aux média pour justifier la similitude des perceptions des risques exprimées par des individus portugais, macanéens, français, et américains, ainsi que la dissimilitude entre ces perceptions et celles d'individus hongrois et russes relevées antérieurement : " alors que les premiers ont un accès illimité au même pool d'informations, les seconds avaient jusqu'à récemment un accès très limité à l'information » (ibid., p. 162).

Enfin, trois études récentes menées par Mullet ont rendu compte de leurs résultats en alléguant l'influence des média sur le risque perçu. Pour Mullet, Lazreg, Candela et Neto (2005), l'équivalence des perceptions des risques exprimées par des étudiants finnois, norvégiens, et suédois témoigne d'une « manière scandinave » de percevoir les risques, largement due à la communication uniforme de ces derniers dans les média scandinaves et à la spécificité scandinave de mettre l'accent sur ce qui se pas- se hors du pays (Nyland, 1993). Pour Macri et Mullet (non publié), c'est l'exposition aux média qui explique la supériorité des estimations de leurs participants français comparées à celles de Grecs, à propos des risques posés par les amphétamines, l'amiante, ou le retraitement nucléaire, thèmes hautement sujets à la publicité en France, et pas en Grèce. De la même façon, Ahmed et al. (2006) rendent compte des perceptions des risques plus élevées des Français comparées aux Égyptiens à propos de questions de santé et d'environnement en évoquant le fait que ces préoccupations ont été beaucoup moins mises en avant dans les média égyptiens que dans les média européens et notamment français.

Ainsi, il s'avère que l'influence des média sur le risque perçu est une hypothèse plus que probable pour rendre compte des similitudes et des différences de perception des risques (cf. Ahmed et al., 2006 ; Macri et Mullet, non publié, pour une synthèse).

\section{Influence des visions du monde}

Selon Lai et Tao (2003, p. 670), « une compréhension de la manière dont les individus pensent ou interprètent les risques ne peut pas être atteinte sans prendre en compte le contexte socioculturel dans lequel les risques se développent et sont discutés ».

C'est en partant de ce postulat que Douglas et Wildavsky (1982) ont mis au point la Théorie Culturelle de la perception des risques, initiée à partir de la conceptualisation « Grid et Group »- quadrillage et groupe - de Douglas (1978). Selon cette conceptualisation, le quadrillage et le groupe sont des coordonnateurs universels aptes à "mesurer " les structures sociales. Le groupe est un cadre environnemental évident. Le quadrillage correspond à un ensemble de règles qui assujettit les individus au cours de leurs interactions et qui définit les divers rôles sociaux. À partir de la combinaison de ces deux éléments, la Théorie Culturelle a formulé l'existence de quatre grands types de biais culturels ou valeurs et croyances intériorisées : la hiérarchie (groupe fort et quadrillage élevé), l'individualisme (groupe faible et quadrillage inexistant), l'égalitarisme (groupe fort et quadrillage inexistant) et le fatalisme (groupe faible et quadrillage élevé). Ces quatre grands biais culturels, combinés aux relations sociales des individus, forment le concept crucial de cette théorie, celui de vision du monde.

Selon cette théorie, la perception des risques est socialement déterminée, culturellement biaisée, c'està-dire influencée par les visions du monde auxquel- 
les adhèrent les individus, ces dernières étant qualifiées de « dispositions orientantes " par Dake (1991). Précisément, Dake $(1990,1991)$ et Wildavsky et Dake (1990) ont mis en évidence que (a) les égalitaires préfèrent un monde aux ressources équitablement distribuées, craignent particulièrement les risques technologiques, conçoivent la nature comme fragile, et n'ont pas confiance en la société ; (b) les hiérarchiques aiment une société organisée, craignent beaucoup les risques de déviance sociale mais peu les risques technologiques, et ont confiance en la société ; (c) les individualistes aiment agir librement et sans contrainte, craignent les risques de guerre, acceptent les risques technologiques, et ont confiance en la société de marché ; (d) les fatalistes tendent à penser que ce qui arrive est réglé d'avance.

Seulement, malgré les efforts des partisans de la Théorie Culturelle consacrés à démontrer sa pertinence pour expliquer la perception des risques, cette théorie présente de nombreuses faiblesses, et

- testée empiriquement - elle explique peu le niveau de risque perçu (Boholm, 1996 ; Sjöberg, 1997, 2000a, 2002). Précisément, Nyland (1993) a montré que les résultats issus de l'application de la Théorie Culturelle souffraient d'un manque de fiabilité, de corrélations faibles et peu significatives entre les visions du monde et la perception des risques, et d'un manque de pouvoir prédictif de ces visions $\mathrm{du}$ monde quant aux estimations de risques. De manière similaire, Sjöberg $(1996 b, 2003)$ ou Marris, Langford et O'Riordan (1998) ont mis en évidence que les visions du monde de la Théorie Culturelle expliquaient seulement autour de $5 \%$ de variance du risque perçu. Un récent travail à grande échelle effectué en France par Brenot, Bonnefous et May (1996) confirme ce résultat.

La Théorie Culturelle apparaît donc être «une théorie qui n'explique qu'une très petite part du phénomène qu'elle étudie et donc présente un intérêt marginal » (Sjöberg, 2003, p. 205). " Il est très improbable que de telles tendances générales (les visions du monde) existent; Ou, si elles existent, il paraît improbable qu'elles aient une influence majeure sur le risque perçu » (Sjöberg, 2003, p. 207).

\section{Influence de l'anxiété}

L'anxiété peut être définie comme une tendance excessive à percevoir des menaces imminentes provenant de l'environnement. Alors que cette façon de définir l'anxiété semble largement partagée, il n'en est rien concernant la façon de mesurer l'anxiété. En effet, les recherches développées ci-dessous - dont l'objectif a été de tester l'influence de l'anxiété sur le risque perçu - ont toutes utilisé une mesure différente de l'anxiété, et ont par conséquent évalué différents types d'anxiété.

Sjöberg (1998b) a travaillé sur l'anxiété par le biais de mesures du sentiment d'inquiétude, sentiment qui se réfère à une réaction émotionnelle selon l'auteur. Après avoir recueilli les perceptions d'un échantillon de suédois quant aux risques posés par diverses substances, activités, et technologies, ces perceptions des risques ont été mises en relation avec deux indices d'inquiétude - un niveau d'inquiétude générale et des inquiétudes plus spécifiées. Les résultats ont montré que ces deux indices d'inquiétude étaient seulement modestement reliés au risque perçu et expliquaient peu les variations de risque perçu associé à chaque item, ce qui a permis à l'auteur de conclure en disant que « les niveaux d'inquiétude et de risque perçu étaient largement statistiquement indépendants » (ibid., p. 91).

Une brève revue de littérature a permis à Bouyer, Bagdassarian, Chaabanne et Mullet (2001) de constater l'inconsistance des résultats concernant la relation entre une mesure du niveau d'anxiété (l'anxiété durable) et le niveau de risque associé aux inondations et aux séismes. Pour clarifier, enrichir, et rendre plus précise la relation potentielle entre l'anxiété et le risque perçu, ces auteurs ont décidé de considérer (a) une vaste taxonomie de 141 risques et (b) deux indices d'anxiété tirés de l'inventaire de Spielberger, Gorsuch et Lushene (1970) - l'anxiété durable (ou anxiété-trait) et l'anxiété transitionnelle (ou anxiétéétat). Après avoir regroupé les estimations relatives aux 141 risques en dix catégories, les auteurs ont pu montrer que les deux indices d'anxiété influençaient significativement mais modestement le niveau de risque perçu associé à cinq des dix catégories identifiées (les niveaux de risques associés aux polluants, activités d'extérieur, transports publics et risques ordinaires ont été influencés par le niveau d'anxiété transitionnelle; le niveau de risques associé aux médicaments psychotropes a été influencé par le niveau d'anxiété durable). Ces résultats ont fait dire à Bouyer et al. (2001, p. 464) que « les niveaux de risques perçus étaient seulement influencés de façon secondaire par les états d'anxiété transitionnelle et plus durable ».

Pour examiner la relation entre l'anxiété et la perception des risques, Källmén (2000) s'est servi de l'échelle d'Anxiété Manifeste (Taylor, 1953), constituée de questions relatives à l'anxiété, à la nervosité, et à différents symptômes somatiques d'an- 
xiété. Contrairement aux deux études évoquées précédemment, les résultats de cette recherche ont montré que la variable anxiété manifeste a expliqué à elle seule près de $30 \%$ de la variance du risque personnel et environ $20 \%$ de la variance du risque global.

Récemment, Kobbeltvedt et al. (2004) ont mesuré le risque dans un contexte militaire. Afin d'étudier la relation entre l'anxiété et la perception des risques, ils ont employé une mesure d'anxiété anticipée définie par eux comme un sentiment qui - sur la base de certains processus cognitifs - est attendu être ressenti dans le futur. Pour remplir cet objectif, leurs participants ont dû estimer 28 incidents critiques tirés de l'échelle C.I.M. (Critical Incidents during Missions, Solberg, 1997) selon les risques personnels et globaux représentés par ces incidents, et selon l'anxiété anticipée ressentie face à ces incidents. En accord avec Kallmén (2000), les résultats de cette étude ont révélé que la variable anxiété anticipée était un déterminant puissant des niveaux de risque personnel et global, puisqu'elle expliquait entre $5 \%$ et $40 \%$ de la variance du risque perçu.

De façon globale, les conclusions tirées par Sjöberg (1998b) ou Bouyer et al. (2001) quant à la faible influence de l'anxiété sur le risque perçu s'opposent donc aux conclusions tirées par Källmén (2000) ou Kobbeltvedt et al. (2004) quant à la forte influence de l'anxiété sur le risque perçu. Cet état de fait semble largement dû aux divergences entre les auteurs concernant leurs choix théoriques à propos de la définition de l'anxiété et leurs choix méthodologiques à propos de l'instrument de mesure de l'anxiété. Autrement dit, les résultats obtenus semblent largement dépendants du type d'anxiété mesuré et de la façon dont il l'a été. Avant de poursuivre les travaux visant à déterminer l'influence de l'anxiété sur le risque perçu, il semble important d'améliorer la compréhension de ce qu'est l'anxiété (Sjöberg, 1998b).

\section{Influence de la confiance sociale}

Selon Siegrist, Cvetkovitch et Roth (2000), la confiance sociale est définie par la volonté de se fier aux institutions et/ou organismes qui ont la responsabilité pour prendre des décisions et agir pour gérer les technologies, l'environnement, et tout autre domaine de la santé et de la sécurité publiques.

À l'image des études sur l'anxiété, les recherches concernant l'influence de la confiance sociale sur le risque perçu font part de résultats contradictoires.

D'une part, Slovic $(1993,2000)$ affirme qu'une des principales raisons pour lesquelles la perception des risques du public est élevée - notamment à propos des technologies - est la confiance sociale, ou plus exactement le manque de confiance. Deux recherches de Siegrist sont en accord avec cette affirmation. Dans une première étude menée à Zurich par Siegrist et al. (2000), les résultats ont en effet montré que la confiance sociale accordée par les individus aux institutions responsables - née du sentiment des premiers que ces dernières partagent les mêmes valeurs qu'eux et sont donc socialement proches d'eux - était un déterminant clé du risque perçu : les étudiants confiants ont estimé les risques associés aux pesticides, à l'énergie nucléaire et aux édulcorants comme plus faibles que ceux peu confiants. Dans une deuxième étude où 91 américains ont estimé diverses activités et technologies, Siegrist et Cvetkovitch (2000) ont précisément établi les conditions d'influence de la confiance sur le risque perçu. En fait, c'est quand les profanes n'ont pas de connaissances détaillées nécessaires à l'estimation des risques présentés par telle ou telle technologie qu'ils se fient aux opinions des experts et/ou des autorités gestionnaires de ces technologies pour juger. En revanche, lorsqu'ils estiment avoir des connaissances adéquates pour estimer les risques, alors ils effectuent leurs propres jugements sans se fier aux opinions des autres : les connaissances personnelles viennent donc médiatiser l'influence de la confiance sociale sur les jugements de risque.

D'autre part, Sjöberg (1998c, p. 9) affirme que « l'importance de la confiance sociale sur le risque perçu est limitée ». À partir de données suédoises concernant des indices de confiance générale - comme la confiance dans les politiques de manière globale, la confiance dans l'industrie en général, ou la confiance dans l'honnêteté générale des individus - Sjöberg (1999, 2001) et Sjöberg et af Wahlberg (2002) ont en effet montré que ces indices de confiance générale expliquaient très modérément le niveau de risque perçu (à hauteur de $10 \%$ de la variance du risque perçu associé au nucléaire et de $5 \%$ de la variance du risque perçu associé à diverses activités et technologies). Une fois publiés, ces résultats ont fait l'objet de vives critiques de la part de Siegrist et Cvetkovich (2000). Selon eux, le faible pouvoir explicatif de la confiance sociale mis en évidence par les travaux de Sjöberg n'est pas très surprenant et pas d'un très grand intérêt pratique ou théorique étant donné le manque de spécificité des indices de confiance choisis. Autrement dit, ils affirment que le pouvoir explicatif de la confiance sociale ne peut être correctement mesuré et s'avérer important que si les indices de confiance choisis sont en rapport 
étroit avec la nature des risques considérés. Pour répondre à cette critique, Sjöberg $(2000 b, 2001)$ a interrogé des participants suédois en considérant des indices spécifiques de confiance sociale. Ces indices ont expliqué environ $20 \%$ de la variance du risque perçu posé par le nucléaire et $10 \%$ de la variance du risque perçu posé par diverses activités et technologies (Sjöberg, 2001). Ainsi, même s'il concède que les mesures de confiance ont besoin d'être spécifiées pour rendre compte plus efficacement du risque perçu, Sjöberg (2000b, p. 170) affirme tout de même que « la confiance sociale est un déterminant [seulement] modérément important dans la perception des risques ».

Finalement, en considérant l'ensemble de ces résultats, tous les auteurs s'accordent pour dire que l'influence de la confiance sociale sur le risque perçu est largement dépendante de la façon dont elle est spécifiée. Malgré tout, alors que les travaux de Siegrist et ses collaborateurs montrent une forte influence d'indices spécifiques de confiance sociale sur le risque perçu, ceux menés par Sjöberg montrent une influence modérée. La nature des échantillons interrogés (étudiants américains ou suisses vs profanes représentatifs de la population suédoise) peut être à l'origine d'une partie de ces différences.

\section{Influence des croyances religieuses}

Les recherches qui se sont intéressé à l'influence potentielle des croyances religieuses sur le risque perçu ont adopté deux démarches : soit elles ont cherché à identifier l'effet du degré d'engagement religieux sur le risque perçu, soit elles ont plus spécifiquement interrogé l'effet de divers types de croyances sur le risque perçu.

Une étude caractéristique de la première démarche - conduite par Sjöberg et af Wahlberg (2002) - a mesuré le degré d'engagement religieux de 151 profanes suédois grâce à l'échelle SROS II (Swedish Religious Orientation Scale version 2, Hovemyr, 1996). Les résultats ont mis en évidence une corrélation positive consistante entre la quête religieuse (c'est-à-dire le fait d'avoir « la foi » et de s'en servir pour définir le sens de la vie) et la perception des risques, indiquant que les scores de quête religieuse expliquaient $10 \%$ de la variance du risque perçu.

La deuxième démarche a récemment fait l'objet de plusieurs études. Parmi elles, celle de Kouabenan (1998, p. 243) a testé la supposition selon laquelle « la persistance profondément enracinée de certaines croyances et pratiques religieuses animistes peut mener à des erreurs systématiques de jugements de risques ». 553 participants ivoiriens ont rempli un questionnaire de risques d'accidents de la route et un questionnaire de croyances dans le destin exprimant un certain niveau de fatalisme ou de superstition. Les résultats ont montré que les individus fatalistes avaient une connaissance limitée des risques des accidents, ce qui les menait à en surestimer certains et à en sous-estimer certains autres : les croyances fatalistes influencent donc la perception des risques des accidents. Plus récemment, Sjöberg et af Wahlberg (2002) ont étudié de façon empirique les relations potentielles entre la perception des risques et les croyances de type New Age, mesurées principalement grâce à l'Echelle RPBS (Revised Paranormal Belief Scale, Tobacyk, 1988). Une fois regroupées en quatre facteurs - intitulés Croyances en une conscience plus élevée, Croyance en la réalité de l'âme, Déni de la connaissance analytique, et Superstition populaire traditionnelle - les croyances New Age se sont avérées être corrélées au risque perçu du nucléaire, et notamment le premier facteur. Considérés ensemble, ces facteurs New Age ont expliqué près de $15 \%$ de la variance du risque perçu du nucléaire. Ce résultat a depuis été confirmé par Sjöberg (2003, 2004).

De façon synthétique, ces études montrent que l'adhésion à des croyances religieuses a un impact sur la perception des risques et semble accroître le niveau de risque perçu. Concernant les croyances New Age, Sjöberg et af Wahlberg (2002) concluent d'ailleurs qu'elles peuvent être un composant important du risque perçu associé aux nouvelles technologies en général et au nucléaire en particulier.

\section{Influence de la personnalité}

Selon Rolland (2004), le concept de personnalité renvoie à un ensemble dynamique et relativement cohérent, systématique et stable de traits qui sont des tendances à générer des ensembles structurés de pensées (cognitions), d'affects et d'actions qui font d'un individu donné une personne unique. Aujourd'hui, d'après Digman (1996), le modèle le plus répandu et le plus consensuel pour rendre compte de la personnalité est le «Big Five», à savoir une structure hiérarchique constituée de cinq grands facteurs - Extraversion, Agréabilité, Caractère Consciencieux, Stabilité émotionnelle (ou Neuroticisme) et Intellect (ou Ouverture à l'expérience) - qui tendent à décrire de façon parcimonieuse et exhaustive les nombreuses différences individuelles. Etant donné l'expansion de ce modèle, 
les recherches présentées ci-dessous - dont l'objectif a été de caractériser l'effet potentiel de la personnalité sur le risque perçu - ont été pour la plupart conduites selon ce modèle de Big Five.

À partir d'estimations d'étudiants zurichois, Vollrath, Knoch et Cassano (1999) ont pu décrire les relations entre la personnalité et la sensibilité perçue aux risques liés aux comportements comme fumer, boire, conduire après avoir bu, avoir des relations sexuelles risquées. Ils ont notamment montré que les individus ayant eu des scores élevés sur les dimensions d'Agréabilité et de Caractère Consciencieux ont exprimé des perceptions des risques plus élevées que ceux n'ayant pas ces profils de personnalité.

Plusieurs études conduites par Sjöberg se sont elles aussi intéressé à l'effet de la personnalité sur la perception des risques. À l'aide de l'échelle MPI développée par Eysenck (Maudsley Personality Inventory, Eysenck, Eysenck et Barett, 1985), Sjöberg et af Wahlberg (2002) ont notamment pu montrer que le facteur de personnalité Neuroticisme était corrélé à .23 avec le risque perçu du nucléaire, c'est-à-dire que les individus neurotiques percevaient plus de risques issus du nucléaire que les autres. À l'aide de deux autres instruments de personnalité en version suédoise - le MBTI (Myers-Briggs Type Indicator, Mardberg, Nienmaa, Hillström et Carlstedt, 1994) et le Big Five (Hendruks, 1997) - Sjöberg (2003) a confirmé et étendu ce premier résultat : il semble que les individus instables émotionnellement, c'est-à-dire neurotiques, perçoivent les risques posés par diverses activités et technologies de façon plus élevée que les autres.

Même si ces études ont montré un effet de la personnalité sur la perception des risques, celui-ci est apparu être soit modéré (Sjöberg, 2003; Sjöberg et af Wahlberg, 2002), soit consistant et assez élevé mais établi sur un type très limité de risques (Vollrath et al., 1999). Autrement dit, la perception des risques n'est pas facile à expliquer avec des concepts de personnalité, même s'il semble que d'autres constructs de personnalité peuvent avoir un effet bien plus conséquent sur le risque perçu (Sjöberg, 2003; af Wahlberg, 2001). C'est en se basant sur ces dernières déclarations que Chauvin, Hermand et Mullet (à paraitre) ont testé l'effet de la personnalité sur la perception des risques sous un angle inédit. Au lieu des Big Five, ils ont considéré les facettes associées à chaque Big Five - à savoir les 9 constituants de chacun des 5 grands facteurs - proposées par l'IPIP (International Personality Item Pool, Goldberg, 1999). Cette analyse plus fine a permis aux auteurs d'identifier les facettes de personnalité « clés », c'est-à-dire les facettes les plus déterminan- tes du niveau de risque perçu, intitulées Révélation de soi, Altruisme et Tendresse, Rationalité et Efficacité, Modération et Tranquillité, et Créativité, Imagination et Réflexion. Selon les risques considérés, l'une ou l'autre de ces facettes a expliqué de $4 \%$ à $14 \%$ de la variance du risque perçu.

À l'image de l'influence des croyances New Age, il semble qu'une prise en compte pertinente de la personnalité peut avoir un effet non négligeable sur le risque perçu.

La seconde catégorie d'études développées dans cette deuxième partie vient compléter la première catégorie évoquée plus haut dans cette revue de question. Précisément, suite au constat dressé par la première série de travaux selon lequel il existe des différences de perception des risques entre les individus selon diverses variables sociodémographiques (comme le genre ou l'âge), la seconde série de travaux a tenté d'identifier l'origine de ces différences, en testant divers déterminants psychosociaux du risque perçu.

De façon synthétique, les divers déterminants testés ont été plus ou moins efficaces pour rendre compte du risque perçu. Précisément, les études évoquées ont montré que (a) les visions du monde rendent très peu compte du niveau de risque perçu et semblent donc présenter un intérêt mineur pour expliquer ce phénomène ; (b) l'anxiété, la confiance sociale, les croyances religieuses et la personnalité expliquent modérément le niveau de risque perçu; (c) le statut/position des individus dans la société et l'exposition médiatique sont des déterminants importants du risque perçu.

\section{Conclusion}

En faisant sienne la définition de Slovic et al. (1982) selon laquelle «étudier la perception des risques consiste à examiner les opinions que les individus expriment lorsqu'ils doivent évaluer des technologies, substances ou activités plus ou moins risquées » (ibid., p. 83), cette recherche s'inscrit clairement dans une démarche bipolaire concernant l'étude du risque perçu, et distingue ainsi deux objectifs différents et complémentaires: (a) l'étude des risques en eux-mêmes et (b) l'étude des individus percevants. Autrement dit, plus concrètement, cette recherche a d'emblée proposé de synthétiser les objectifs des travaux sur le risque perçu en deux questions principales : (a) Pourquoi certaines activités, substances, ou technologies sont-elles perçues plus risquées que d'autres par la population en général ?; (b) Pourquoi 
certains individus perçoivent-ils un ensemble d'activités, substances ou technologies plus risquées que d'autres individus?

Concernant la première de ces deux questions, de nombreuses recherches conduites essentiellement selon le paradigme psychométrique (Slovic et al., 1985) ont proposé une réponse claire et satisfaisante : les déterminants principaux à l'origine du caractère perçu plus risqué de certaines activités par rapport à d'autres sont le sentiment de peur inspirée par un risque et le manque de connaissance associée à un risque (cf. par exemple, Bronfman et Cifuentes, 2003 ; Cha, 2000 ; Mullet et al., 1993 ; Slovic et al., 1979, 1980, 1985).

En revanche, concernant la deuxième question, les réponses apportées ont été plus confuses et restent insuffisantes. De nombreuses dimensions personnelles ont été considérées avec plus ou moins de succès. Dans un souci de clarification, cette revue de littérature a été entreprise et a été consacrée aux études qui ont tenté de comprendre - c'est-à-dire décrire et/ou expliquer - pourquoi certains individus perçoivent un ensemble d'activités, substances ou technologies plus risquées que d'autres individus. Ce travail a donc traité de l'influence des variables distales sur la perception des risques, en prenant en compte à la fois des variables individuelles distales de nature sociodémographique et des variables individuelles distales de nature psychosociale.

Dans un premier temps, cette recherche s'est focalisée sur la catégorie rassemblant les travaux descriptifs relatifs aux variables sociodémographiques, qui ont visé à évaluer si les estimations effectuées par tel type d'individus étaient significativement différentes de celles effectuées par tel autre type d'individus. Globalement, ces travaux ont montré que (1) les femmes ont une perception des risques plus élevée que celles des hommes ; (2) l'effet de l'âge sur le risque perçu reste équivoque; (3) les individus possédant un haut niveau d'expertise scientifique perçoivent les risques de manière plus faible que les autres ; (4) les individus moins instruits sont plus sensibles aux risques que ceux mieux instruits ; (5) la profession et l'expérience jouent un rôle important dans la perception des risques; (6) les individus les plus pauvres ont les perceptions des risques les plus élevées ; (7) les orientations politiques semblent compter dans la perception des risques des individus ; (8) les jugements des risques sont différents selon le point de vue adopté par les individus quant à la cible visée.

Dans un deuxième temps - afin de compléter les résultats descriptifs mis en évidence par la première catégorie de travaux et donc offrir une caractérisation plus optimale du risque perçu par les individus - cette étude a considéré une deuxième catégorie de travaux, dont l'objectifa été de proposer des explications aux différences évoquées ci-dessus à l'aide de diverses variables psychosociales. Globalement, ces travaux ont montré que les divers déterminants testés pouvaient être regroupés en trois classes, selon leur efficacité à rendre compte du risque perçu. La première classe - constituée de la variable Visions du monde - fait part d'un déterminant clairement peu efficace pour expliquer le niveau de risque perçu. Dans sa forme actuelle, ce concept a en effet très peu d'influence sur le risque perçu. La deuxième classe - composée des variables prometteuses Anxiété, Confiance sociale, Croyances religieuses et Personnalité - rassemble des déterminants qui semblent avoir une certaine efficacité pour expliquer le niveau de risque perçu. Toutefois, un long travail préalable théorique et méthodologique paraît nécessaire avant d'aboutir à une prise en considération uniforme et pertinente de ces variables. Enfin, la troisième classe - formée des « valeurs sûres » Statut/position des individus dans la société et Exposition médiatique - réunit des déterminants clairement efficaces pour expliquer le risque perçu.

Considérées ensemble, ces deux séries de travaux ont donc offert une riche caractérisation de la perception des risques. Toutefois, malgré le double éclairage fourni par cette revue de littérature, il reste beaucoup à connaître des perceptions des risques exprimées par les individus. En effet, la part descriptive et la part explicative apportées par les variables distales considérées - sociodémographiques et psychosociales - semblent partielles et nécessitent d'être complétées, afin de répondre de façon pleine et appropriée à la question de savoir pourquoi certains individus jugent un ensemble de substances, activités ou technologies plus risquées que d'autres individus. 


\section{RÉFÉRENCES}

- Ahmed R. A., Macri D. et Mullet E. (2006): Societal risk perception among a sample of Egyptian adolescents and young adults. The Journal of North African Studies, Vol. 11, N³, pp. 323-334.

- Barke R. P., Jenkins-Smith H. et Slovic P. (1997): Risk perceptions of men and women scientists. Social Science Quaterly, Vol. 78, $\mathrm{N}^{\circ} 1$, pp. 167-176.

- Barny M. H., Bonnefous S. et Brenot J. (1992): Données sur les déchets radioactifs. Évolutions depuis 1977 (Note LSEES No 92/36). Centre d'Etudes Nucléaires de Fontenay-aux-Roses.

- Bastide S., Moatti J.-P., Pages J.-P. et Fagnani F. (1989): Risk perception and social acceptability of technologies : The French case. Risk Analysis, 9(2), pp. 215-223.

- Baumer T. L. (1978): Research on fear of crime in the United States. Victimology, 3, pp. 254-264.

- Benthin A., Slovic P. et Severson H. (1993): A psychometric study of adolescents' risk perception. Journal of Adolescence, 16, pp. 153-168.

- Blomkvist A. C. et SJöberg L. (1987): Communication about risks. In L. Sjöberg (Dir.), Risk and Society. Londres, Allen \& Unwin.

- Boноlм A. (1996): Risk perception and social anthropology : critique of Cultural Theory. Ethnos, Vol. 61, N¹-2, pp. 64-84.

- Boholm A. (1998): Comparative studies of risk perception: A review of twenty years of research. Journal of Risk Research, Vol. 1, N², pp. 135-163.

- Bouyer M., Bagdassarian S., Chaabane S. et Mullet E. (2001): Personality correlates of risk perception. Risk Analysis, 21, pp. 457465.

- Brenot J., Bonnefous S. et Mays C. (1996): Cultural Theory and risk perception: Validity and utility explored in the French context. Radiation Protection Dosimetry, 68, pp. 239-243.

- Bronfman N. C. et Cifuentes L. A. (2003): Risk perception in a developing country: The case of Chile. Risk Analysis, Vol. 23, N 6 , pp. 1271-1285.

- Cadet B. et Kouabenan D. R. (2005): Assessing and modelling risks: Contributions and limits of several paradigms in security diagnosis. Travail Humain, Vol. 68, N¹, pp. 7-35.

- Causse P., Kouabenan D. R. et Delhomme P. (2004): Alcohol-related accident risk perception by young drivers: Some determinants of comparative optimism. Travail Humain, Vol. 67, N³, pp. 235-256.

- Chн Y. J. (2000): Risk perception in Korea: An application of psychometric paradigm. International Journal of Risk Assessment and Management, Vol. 1, N¹-2, pp. 42-51.

- Chauvin B., Hermand D. et Mullet E. (à paraître): Risk perception and personality facets. Risk Analysis.

- Cohn L. D., Macfarlane S., Yanez C. et Imai W. K. (1995): Risk perception: Differences between adolescents and adults. Health Psychology, Vol. 14, N³, pp. 217-222.

- Combs B. et Slovic P. (1979): Newspaper coverage of causes of death. Journalism Quaterly, 56, pp. 837-843, 849.

- DAKE K. (1990): Technology on trial: Orienting dispositions toward environmental and health hazards. Thèse de doctorat, manuscrit non publié, Berkeley, University of California.

- DAKE K. (1991): Orienting dispositions in the perception of risk. Journal of Cross-Cultural Psychology, 22, pp. 61-82.

- Digman J. M. (1996): The curious history of the Five-Factor Model. In J. S. Wiggins (Dirs.), The Five-Factor Model of personality: Theoretical perspective. New York, Guilford Press.

- Douglas M. (1978): Cultural Bias. Royal Anthropological Institute of Great Britain and Ireland, Occasional papers, 35.

- Douglas M. et Wildavsky A. (1982): Risk and Culture. Berkeley, University of California Press.

- Dupont R. L. (1980): Nuclear phobia: Phobic thinking about nuclear power. Washington, The Media Institute.
- Eiser J. R., Hannover B., Mann L., Morin M., van der Pligt J. et WeBLey P. (1990): Nuclear attitudes after Chernobyl: A cross national study. Journal of Environmental Psychology, 10, pp. 101-110.

- Englander T., Farago K., Slovic P. et Fischioff B. (1986): Comparative analysis of risk perception in Hungary and the United States. Social Behavior, 1, pp. 55-66.

- Eysenck S. B, Eysenck H. J. et Barrett P. (1985): A revised version of the Psychoticism scale. Personality and Individual Differences, 6, pp. 2129.

- Finucane M. L., Slovic P., Mertz C. K., Flynn J. et Sattterfield T. A. (2000a): Gender, race, and perceived risk: The "White male" effect. Health, Risk and Society, Vol. 2, N², pp. 159-172.

- Fischioff B., Watson S. et Hope C. (1984): Defining risk. Policy Sciences, 17, pp. 123-139.

- Fiske S. T. (1987): People's reactions to nuclear war: Implications for Psychologists. American Psychologist, 42, pp. 207-217.

- Flynn J., Slovic P. et Mertz C. K. (1994): Gender, race, and perception of environmental health risks. Risk Analysis, Vol. $14, \mathrm{~N}^{\circ} 6$, pp. 1101-1108.

- Freudenburg W. R. (1988): Perceived risk, real risk: Social science and the art of probabilistic risk assessment. Science, 242, pp. 44-49.

- Frewer L. J., Shepherd R. et Sparks P. (1993): The interrelationship between perceived knowledge, control and risk associated with a range of food-related hazards targeted at the individual, other people and society. Journal of Food Safety, 14, pp. 19-40.

- Goldberg L. R. (1999): A broad-bandwidth, public-domain, personality inventory measuring the lower-level facets of several FiveFactor models. In I. Mervielde, I. Deary, F. De Fruyt et F. Ostendorf (Dirs.), Personality Psychology in Europe. Tilburg, The Netherlands, Tilburg University Press.

- Goszczynska M., Tyszka T. et Slovic P. (1991): Risk perception in Poland: A comparison with three other countries. Journal of Behavioural Decision Making, 4, pp. 179-93.

- Granger Morgan M., Slovic P., Nair I., Geisler D., McGregor D., Fischhoff B., Lincoln D. et Florig K. (1985): Powerline frequency electric and magnetic fields: A pilot study of risk perception. Risk Analysis, Vol. 5, N², pp. 139-149.

- Hendrunks A. A. (1997): The construction of the Five-Factor Personality Inventory (FFPI). Groningen, University Press of Groningen.

- Hermand D., Karsenty S., Py Y., Gulllet L., Chauvin B., Simeone A., Munoz Sastre M. T. et Mullet E. (2003): Risk target: An interactive context factor in risk perception. Risk Analysis, Vol. 23, $\mathrm{N}^{\circ} 4$, pp. 821-828.

- Hermand D., Mullet E. et Rompteaux L. (1999): Societal risk perception among children, adolescents, adults, and elderly people. Journal of Adult Development, Vol. 6, N², pp. 137-143.

- Hovemyr M. (1996): Religious orientation and the attribution of success and failure: An explorative study in different cultural contexts. Thèse de doctorat, manuscrit non publié, Stockholm, Université de Stockholm.

- Källmén H. (2000): Manifest anxiety, general self-efficacy and locus of control as determinants of personal and general risk perception. Journal of Risk research, Vol. 3, N², pp. 111-120.

- Kepowicz-Lazreg C. et Mullet E. (1993): Societal risks as seen by the French public. Risk Analysis, 13, pp.253-258.

- Kobbeltvedt T., Brun W. et Laberg J.C. (2004): Measuring and modelling risk in a natural setting. Journal of Risk Research, Vol. 7, Nº 8, pp. 789-810.

- Koné D. et Mullet E. (1994): Societal risks perception and media coverage. Risk Analysis, 14, pp. 21-24.

- Kouabenan D. R. (1998): Beliefs and the perception of risks and accidents. Risk Analysis, Vol. 18, N³, pp. 243-252.

- Kraus N. N., Malmfors T. et Slovic P. (1992): Intuitive toxicology: Expert and lay judgments of chemical risks. Risk Analysis, Vol. 12, $\mathrm{N}^{\circ}$, pp. 215-232. 
- Lai J. C. et Tao J. (2003): Perception of environmental hazards in Hong Kong Chinese. Risk Analysis, Vol. 23, N4, pp. 669-684

- Lazo J. K., Kinnell J. C. et Fischer A. (2000): Expert and layperson perceptions of ecosystem risk. Risk Analysis, Vol. 20, N², pp. 179193.

- Lichtenstein S., Slovic P., Fischhoff B., Layman M. et Combs B. (1978): Judged frequency of lethal events. Journal of Experimental Psychology, 4, pp. 551-78.

- Macri D. et Mullet E. (non publié): Cross-cultural differences in risk perception: A typological approach.

- Mardberg B., Nienmaa P., Hillstrom B. el Carlstedt B. (1994): Myers-Briggs Type Indicator. Manual. Stockholm, Université de Stockholm.

- Marris C., Langford I. et O'Riordan T. (1998): A quantitative test of the Cultural Theory of risk perceptions: Comparison with the Psychometric Paradigm. Risk Analysis, Vol. 18, N5, pp. 635-347.

- McDaniels T., Axelrod L. J., Cavanagh N. S. et Slovic P. (1997): Perception of ecological risk to water environments. Risk Analysis, Vol. $17, \mathrm{~N}^{\circ} 3$, pp. 341-352.

- McGregor D. G., Slovic P. et Granger Morgan M. (1994): Perception of risks from electromagnetic fields: A psychometric evaluation of a risk communication approach. Risk Analysis, Vol. 14, N5, pp. 815823.

- Mertz C.K., Slovic P. et Purchase I. F. (1998): Judgments of chemical risks: Comparisons among senior managers, toxicologists, and the public. Risk Analysis, Vol. 18, N4, pp. 391-404.

- Mullet E., Ben Bouazza M., Dupont V. et Bertrand A. (1998): Risk perception and energy production. Human and Ecological Risk Assessment, Vol. 4, N¹, pp. 153-175.

- Mullet E., Duquesnoy C., Raiff P., Fahrasmane R. et Namur E. (1993): The evaluative factor of risk perception. Journal of Applied Social Psychology, 23, pp. 1594-1605.

- Mullet E., Lazreg C., Candela C. et Neto F. (2005): The Scandinavian way of perceiving societal risks. Journal of Risk Research, Vol. 8, N¹, pp. 19-30.

- Neil N., Slovic P. et Hakkinen P. J. (1993): Mapping Consumer Perceptions of Risk. Washington, Chemical Manufactures Association.

- Neto F. et Mullet E. (2000): Societal risks as seen by the Portuguese public. European Review of Applied Psychology, Vol. 50, N¹, pp. 155163.

- Neto F. et Mullet E. (2001): Societal risks are seen by Chinese student living in Macao. Journal of Risk Research, Vol. 4, N¹, pp. 6373.

- Nyland L. G. (1993): Risk perception in Brazil and Sweden (RHIZIKON : Risk Research Report $\left.\mathrm{N}^{\circ} 15\right)$. Center for Risk Research, Stockholm School of Economics.

- Orton B. R., Sjöberg L., Jung J., Urge-Vorsatz D. et TamassynéBIRO M. (2001): Risk perception by industrial radiographers: Hungary and the UK compared. Journal of Risk Research, Vol. 4, N¹, pp. 1729.

- Palmer C. G. (2003): Risk perception: Another look at the 'white male' effect. Health, Risk \& Society, Vol. 5, $\mathrm{N}^{\circ} 1$, pp. 71-83.

- Peters E. et Slovic P. (1996): The role of affect and worldviews as orienting dispositions in the perception and acceptance of nuclear power. Journal of Applied Social Psychology, Vol. 26, n¹6, pp. 14271453

- Pilisuk M. et Acredolo C. (1988): Fear of technological hazards : One concern or many? Social Behaviour, 3, pp. 17-24.

- Rolland J.-P. (2004): L'évaluation de la personnalité: le modèle en cinq facteurs. Sprimont, Mardaga.

- SAVAGE I. (1993): Demographic influences on risk perceptions. Risk Analysis, Vol. 13, N4, pp. 413-420.
- Schütz H. et Wiedemann P. M. (1998): Judgments of personal and environmental risks of consumer products. Risk Analysis, Vol. 18, $\mathrm{N}^{\circ} 1$, pp. 119-129.

- Siegrist M. et Cvetrovitch G. (2000): Perception of hazards: The role of social trust and knowledge. Risk Analysis, Vol. 20, $\mathrm{N}^{\circ} 5$, pp. 713-719.

- Siegrist M., Cvetrovitch G. et Roth C. (2000): Salient value similarity, social trust, and risk/benefit perception. Risk Analysis, 20, pp. 353-362.

- SJÖBERG L. (1996a): A discussion of the limitations of the psychometric and cultural theory approaches to risk perception ? Radiation Protection Dosimetry, Vol. 68, N³/4, pp. 219-225.

- SJöberG L. (1996b): Risk perception by politicians and the public (RHIZIKON : Risk Research Report N²6). Center for Risk Research, Stockholm School of Economics.

- SJöberG L. (1997): Explaining risk perception: An empirical and quantitative evaluation of Cultural Theory. Risk Decision and Policy, 2, pp. 113-130.

- SJÖBERG L. (1998a): Worldviews, political attitudes and risk perception. Risk : Health, Safety and Environment, 137, pp. 137-152.

- SJÖBERG L. (1998b): Worry and risk perception. Risk Analysis, Vol. 18, $\mathrm{N}^{\circ} 1$, pp. 85-93.

- SjöbergG L. (1998c): Risk perception: Experts and the public. European Psychologist, Vol. 3, N¹, pp. 1-12.

- SJöвerg L. (1999): Perceived competence and motivation in industry and government as factors in risk perception. In G. Cvetkovich et R.E. Löfstedt (Dirs.), Social trust and the management of risk. Londres, Earthscan.

- SJöberg L. (2000a): Factors in risk perception. Risk Analysis, Vol. 20, $\mathrm{N}^{\circ} 1$, pp. 1-11.

- SJÖBERG L. (2000b): Specifying factors in radiation risk perception. Scandinavian Journal of Psychology, 41, pp. 169-174.

- SJÖBERG L. (2001): Limits of knowledge and the limited importance of trust. Risk Analysis, Vol. 21, Nº1, pp. 189-198.

- SJÖBERG L. (2002): Are received risk perception models alive and well? Risk Analysis, Vol. 22, N4, pp. 665-669.

- SJöBerg L. (2003): Distal factors in risk perception. Journal of Risk Research, Vol. 6, N³, pp. 187-211.

- SJÖBERG L. (2004): Principles of risk perception applied to gene technology. European Molecular Biology Organization Reports, 5, pp. 547551

- Sjöberg L. et Drottz-SJöberg B.M. (1993): Attitudes to nuclear waste (RHIZIKON : Risk Research Report $\mathrm{N}^{\circ} 12$ ). Center for Risk Research, Stockholm School of Economics.

- Sjöberg L., Kolarova D., Rucai A. A. et Bernström M. L. (1996): Risk perception in Bulgaria and Romania (RHIZIKON : Risk Research Report $\left.\mathrm{N}^{\circ} 4\right)$. Center for Risk Research, Stockholm School of Economics.

- SJöberg L. et Torell G. (1993): The development of risk acceptance and moral valuation. Scandinavian Journal of Psychology, 34, pp. 223236.

- Sjöberg L. et af Wahlberg A. (2002): Risk perception and New Age beliefs. Risk Analysis, Vol. 22, N4, pp. 751-764.

- Slovic P. (1987): Perception of risk. Science, 236, pp. 280-285.

- SLovic P. (1992): Perception of risk: Reflections on the psychometric paradigm. In S. Krimsky et D. Golding (Dirs.), Social Theories of Risk. New York, Praeger.

- Slovic P. (1993): Perceived risk, trust, and democracy. RiskAnalysis, 13, pp. 675-682

- SLovic P. (1996): Perception of risk from radiation. Radiation Protection Dosimetry, Vol. 68, N³/4, pp. 165-180.

- SLovic P. (1999): Trust, emotion, sex, politics, and science: Surveying the risk-assessment battlefield. Risk Analysis, 19, pp. 689-701. 
- Slovic P. (2000): Introduction and overview. In P. Slovic (Dir.), The Perception of Risk. Londres, Earthscan.

- Slovic P., Fischioff B. et Lichtenstein S. (1979): Rating the risks. Environment, Vol. 21, N³, pp. 14-20;36-39.

- Slovic P., Fischhoff B. et Lichtenstein S. (1980): Facts and fears: Understanding perceived risks. In R. C. Schwing et W. A. Alberts Jr (Dirs.), Societal risk assessment: How safe is safe enough? New York, Plenum Press.

- Slovic P., Fischhoff B. et Lichtenstein S. (1982): Why study risk perception? Risk Analysis, Vol. 2, N², pp. 83-93.

- Slovic P., Fischioff B. et Lichtenstein S. (1985a): Characterizing perceived risk. In R. Kates, C. Hohenemser, et J. X. Kasperson (Dirs.), Perilous progress: Managing the hazards of technology. Boulder, Westview Press.

- Slovic P., Malmfors T., Krewski D., Mertz C. K., Neil N. et Bartlett S. (1995): Intuitive toxicology. II. Expert and lay judgments of chemical risks in Canada. Risk Analysis, Vol. 15, Nº6, pp. 661-675.

- Solberg O. A. (1997): UN Observers: Stress and reactions among Norwegian UN observers in the former Republic of Yugoslavia. Thèse de doctorat, manuscrit non publié, Trondheim, Norvège.

- Spielberger C. D., Gorsuch R. L. et Lushene R. E. (1970): Manual for the State-Trait Anxiety Inventory. Palo Alto, Consulting Psychologist Press.

- Steger M. A. et WitT S. L. (1989): Gender differences in environmental orientations: A comparison of publics and activists in Canada and the U.S. West Political Quarterly, 42, pp. 627-649.

- TAYlor A. (1953): A personality scale of manifest anxiety. Journal of Abnormal and Social Psychology, 48, pp. 285-90.
- Tовасук J. J. (1988): A revised paranormal belief scale. Ruston, Louisiana Tech University.

- Vaughan E. et Nordenstam B. (1991): The perception of environmental risks among ethnically diverse groups. Journal of CrossCultural Psychology, Vol. 22, N¹, pp. 29-60.

- Vollrath M., Knoch D. et Cassano L. (1999): Personality, risky health behaviour, and perceived susceptibility to health risks. European Journal of Personality, Vol. 13, $\mathrm{N}^{\circ} 1$, pp. 39-50.

- AF Wahlberg A. E. (2001): The theoretical features of some current approaches to risk perception. Journal of Risk Research, Vol. 4, N³, pp. $237-250$

- Weinstein N. D. (1980): Unrealistic optimism about future life events. Journal of Personality and Social Psychology, Vol. 39, N5, pp. 806-820.

- Weinstein N. D. (1987): Unrealistic optimism about susceptibility to health problems: Conclusions from a community-wide sample. Journal of Behavioral Medicine, Vol. 10, N5, pp. 481-500.

- Weinstein N. D., Lyon J. E., Rothman A. J. et Cutte C. L. (2000): Changes in perceived vulnerability following natural disaster. Journal of Social and Clinical Psychology, Vol. 19, N³, pp. 372-395.

- Wiegman O., Gutteling J. M. et Cadet B. (1995): Perception of nuclear energy and coal in France and the Netherlands. Risk Analysis, Vol. 15, N4, pp. 513-521.

- Wildavsky A. et Dake K. (1990): Theories of risk perception: Who fears what and why? Daedalus, Vol. 119, $\mathrm{N}^{\circ} 4$, pp. 41-60.

- XIE X. F., WAng M. et XU L. C. (2003): What risks are Chinese people concerned about? Risk Analysis, Vol. 23, N4, pp. 685-695.

- ZhaNG J. (1994): Environmental hazards in the Chinese public's eyes. Risk Analysis, Vol. 14, №2, pp. 163-167. 